\title{
Efficient Protein Transduction Method Using Cationic Peptides and Lipids
}

\author{
Kei Yamaguchi, ${ }^{1}$ Mari Inoue, ${ }^{1}$ and Naoki Goshima ${ }^{2}$ \\ ${ }^{1}$ Department of Research and Development, Japan Biological Informatics Consortium (JBiC), 2-4-32 Aomi, Koto-Ku, \\ Tokyo 135-8073, Japan \\ ${ }^{2}$ Biomedicinal Information Research Center, National Institute of Advanced Industrial Science and Technology (AIST), \\ 2-4-7 Aomi, Koto-Ku, Tokyo 135-0064, Japan \\ Correspondence should be addressed to Naoki Goshima, n-goshima@aist.go.jp
}

Received 20 May 2011; Revised 23 July 2011; Accepted 10 August 2011

Academic Editor: Jiing-Kuan Yee

Copyright (๑) 2011 Kei Yamaguchi et al. This is an open access article distributed under the Creative Commons Attribution License, which permits unrestricted use, distribution, and reproduction in any medium, provided the original work is properly cited.

Cationic peptides termed protein transduction domains (PTDs) have been shown to cross biological membranes efficiently. However, proteins transduced by PTDs become entrapped within the endosomal vesicles and are not delivered into organelles. We have developed a novel protein delivery system to enhance the proton sponge effect, which results in rupture of the endosomes, by using a mixture of Wr-T transporter peptide and a commercially available cationic lipid reagent. This peptide and cationic lipid reagent mixture efficiently delivers a variety of cargo proteins into living cells by releasing them from the endosomes.

\section{Introduction}

Protein transduction technology has the potential to constitute a useful tool for studying proteomics. Protein transduction domains (PTDs) such as HIV-1 TAT, pAntp43-58, and polyarginine (R9) are small peptides that are able to transduce a variety of peptides and proteins into several kinds of cells [1-3].

However, protein transduction technology using PTDs has the disadvantage of entrapping the PTD-fused protein within the endosomal vesicles. It has been reported that the main mechanism of protein transduction is the penetration into cells by macropinocytosis; therefore, much of the material becomes entrapped in the macropinosome [4-7]. In fact, Pan et al. published a report on their attempt at reprogramming human fibroblast cells using TAT fusion recombinant proteins, which was unsuccessful even with the help of an endosomal acidification inhibitor, chloroquine and an endosome-disruptive peptide, and hemagglutinin-2 subunit (HA2) [8]. Also, it is reported that methanol fixation causes permeabilization of cell membranes and results in the artificial import of PTD-fused proteins [9].
We focused on developing the transduction technology of proteins using the 30-amino acid peptide/transporter, Wr$\mathrm{T}$, which has an enlarged hydrophobic pocket fused with nine D-enantiomer polyarginines via a Gly-Pro-Gly spacer [10]. To permit the efficient escape of proteins from the endosome, we used cationic lipids to enhance the proton sponge or endosome buffering effect, which is thought to induce osmotic swelling and the consequential rupture of the endosome [11].

In this study, we developed a protein transduction method that can be cultured continuously for adherent living cells using both a functionally reinforced peptide transporter and commercially available cationic lipid reagents.

\section{Materials and Methods}

2.1. Peptide Synthesis, Plasmid Contrast and Reagents. Wr-T peptide was synthesized at Operon Biotechnologies by Fmoc solid-phase peptide synthesis. Crude peptide was purified by reverse-phase high-performance liquid chromatography (purity: $82.6 \%$ ). Peptide identity was confirmed by mass spectrometry. 
VENUS DNA was kindly provided by Dr. A. Miyawaki. Protein expression plasmids were constructed using pEWdestination vectors and a Gateway entry clone by the Gateway LR recombination reaction (Invitrogen Life Technologies). The cationic lipid reagents used for protein transduction included FuGENE6 (Roche Diagnostics), Lipofectamine LTX (Invitrogen Life Technologies) and MultiFectam (Promega) in DNA transfection reagent, and prodeliverIN (OZ Biosciences) and BioPORTER (Genlantis) in protein delivery reagent.

2.2. Expression and Purification of Fusion Proteins. Automated protein in vitro synthesizer Protemist DT (Cell Free Science) synthesized proteins using a wheat germ cell-free system and bilayer reaction. The various expression vectors were transcribed and translated to proteins automatically. Column affinity purification is also conducted available for purifying synthesized GST- or His-tagged fusion proteins by the use of the Protemist DT. Placing Glutathione $4 \mathrm{~B}$ (GE Healthcare) or Ni-sepharose high performance (GE Healthcare) resin in each column, translation reaction mixture was applied to the column. Making wash buffer (GST; Phosphate buffered saline, His; $20 \mathrm{mM}$ Na-phosphate pH7.5, $0.3 \mathrm{M} \mathrm{NaCl}, 20 \mathrm{mM}$ imidazole) pass through the column, purified proteins were eluted by elution buffer (GST; $50 \mathrm{mM}$ Tris- $\mathrm{HCl}, 10 \mathrm{mM}$ reduced glutathione, $\mathrm{pH}$ 8.0, His; $20 \mathrm{mM}$ Na-phosphate pH7.5, 0.3 M NaCl, $500 \mathrm{mM}$ imidazole). The purified proteins confirmed using SDS-PAGE.

2.3. Transduction of Fusion Proteins. HeLa and MRC-5 cells were cultured in DMEM containing 10\% fetal bovine serum (FBS) and antibiotics (100 U/mL penicillin, $100 \mathrm{mg} / \mathrm{mL}$ streptomycin) at $37^{\circ} \mathrm{C}$ in an incubator with $5 \% \mathrm{CO}_{2}$. To determine the intracellular localization of fusion proteins, HeLa cells were first grown in 24-well plates. Then, Wr$\mathrm{T}$ peptide $(3 \mu \mathrm{M})$ and the cargo protein $(1-2 \mu \mathrm{g})$ were mixed in $100 \mu \mathrm{L}$ of PBS at room temperature for $15 \mathrm{~min}$, and then cationic lipid reagents were added as follows: FuGENE6, $1.5 \mu \mathrm{L}$; Lipofectamine LTX, $1 \mu \mathrm{L}$; MultiFectam, $10 \mu \mathrm{L}$; ProdeliverIN, $1.5 \mu \mathrm{L}$; BioPORTER, $2.5 \mu \mathrm{L}$. The solution was incubated at room temperature for $15 \mathrm{~min}$ and overlaid onto the cells, which had been aspirated and washed once with PBS before use. Serum-free medium $(100 \mu \mathrm{L})$ was added, and cells were incubated at $37^{\circ} \mathrm{C}$ in $5 \% \mathrm{CO}_{2}$ for $3 \mathrm{~h}$. Intracellular localization of transduction proteins was visualized in living cells by an inverted fluorescence microscope (Olympus IX51).

2.4. Cell Viability Assay. Cell viability was evaluated with CellTiter-Glo Luminescent Cell Viability Assay (Promega) to determine the number of metabolically active cells in the culture based on quantitation of the ATP present. The reagent was used according to the manufacturer's protocol.

\section{Results and Discussion}

3.1. Properties of Wr-T and Cationic Lipid Reagents for Protein Transduction. A previous study demonstrated that Wr-T induced the intracellular incorporation of cargo protein in fixed cells [12]. To confirm that Alexa-488-labeled BSA protein indeed diffused throughout the cytoplasm, the localization of BSA was compared to that of LysoTracker Red Lysosomal Probe (Lonza) for endocytotic vesicles in living cells. Both BSA and the marker appeared in vesicular structures (Figure 1(a)). No BSA was detected in the cytoplasm, which indicated that it was entrapped within the endosomal vesicles. Therefore, we hypothesized that BSA could escape from endocytotic vesicles if the proton sponge effects of WrT/BSA complexes were increased by the addition of cationic lipids.

To characterize the functions of commercially available cationic lipid reagents for protein transduction, we investigated the localization of BSA in living HeLa and MRC5 cells (Figures 1(b) and S1A of supplementary material available at doi: 10.1155/2011/872065). Wr-T/FuGENE6, Wr-T/ProdeliverIN, and Wr-T/BioPORTER mixtures of BSA appeared in punctuate vesicular structures, and it was suggested that these cationic lipid reagents did not induce the escape of BSA from endosomes. In contrast, we observed dramatic uptake and distribution of BSA throughout the cytoplasm of each HeLa cell when Wr-T/MultiFectam and Wr-T/Lipofectamine LTX mixtures were used. Moreover, BSA was transduced less efficiently by MultiFectam or Lipofectamine LTX used alone. Thus, a mixture of Wr-T and cationic lipid reagents (MultiFectam or Lipofectamine LTX) was required to effectively enhance cell permeability and endosome escape of BSA. Similar results were observed when Alexa-555-labeled ovalbumin protein mixed with Wr-T and cationic lipid reagents were transduced into living cells.

In many cases, protein transduction technology generates toxicity via the transfection carrier. Thus, we compared the degree of toxicity of $\mathrm{Wr}-\mathrm{T}$ and cationic lipid reagents mixtures in living cells. The cationic lipid reagents had low toxicity, while cell viability was increased by the Wr-T and cationic lipid reagents mixture (Figure 1(c)). 3.2. Wr-T and Cationic Lipid Reagents Mixture Effectively
Diffused GST and FLAG Tag-Fused VENUS throughout
the Cytoplasm. We determined whether VENUS proteins
(variants of yellow fluorescent protein) were delivered into
HeLa cells. VENUS expression vectors were used to produce
VENUS protein with a polyhistidine tag at the C-terminus
(3H-VENUS). The soluble fractions of the recombinant
VENUS proteins were purified on a Ni Sepharose column
(Figure 2 (a)). Even with a mixture of Wr-T and cationic
lipid reagents, 3 H-VENUS was poorly delivered into the cells.
Furthermore, we detected aggregate fluorescence, which
might have occurred because 3 H-VENUS, Wr-T and cationic
lipid reagents partly form unstable complexes such as large
aggregates (Figure 2 (b)). It is therefore reasonable to propose
that Wr-T and cationic lipid reagents mixtures would not
be able to deliver all proteins into the cells. Therefore, we
devised a cargo protein fused with glutathione S-transferase
(GST) and FLAG tag to promote stable association and not
agglutination with Wr-T peptide and cationic lipid reagents.
We had previously confirmed that GST and FLAG tag used 

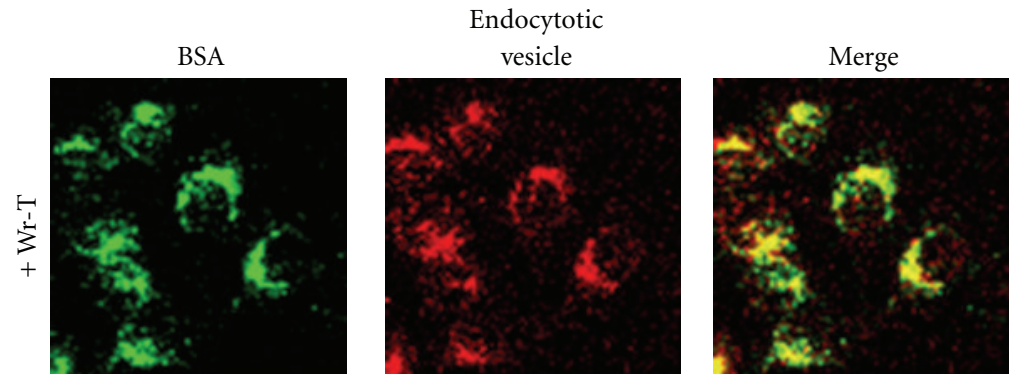

(a)
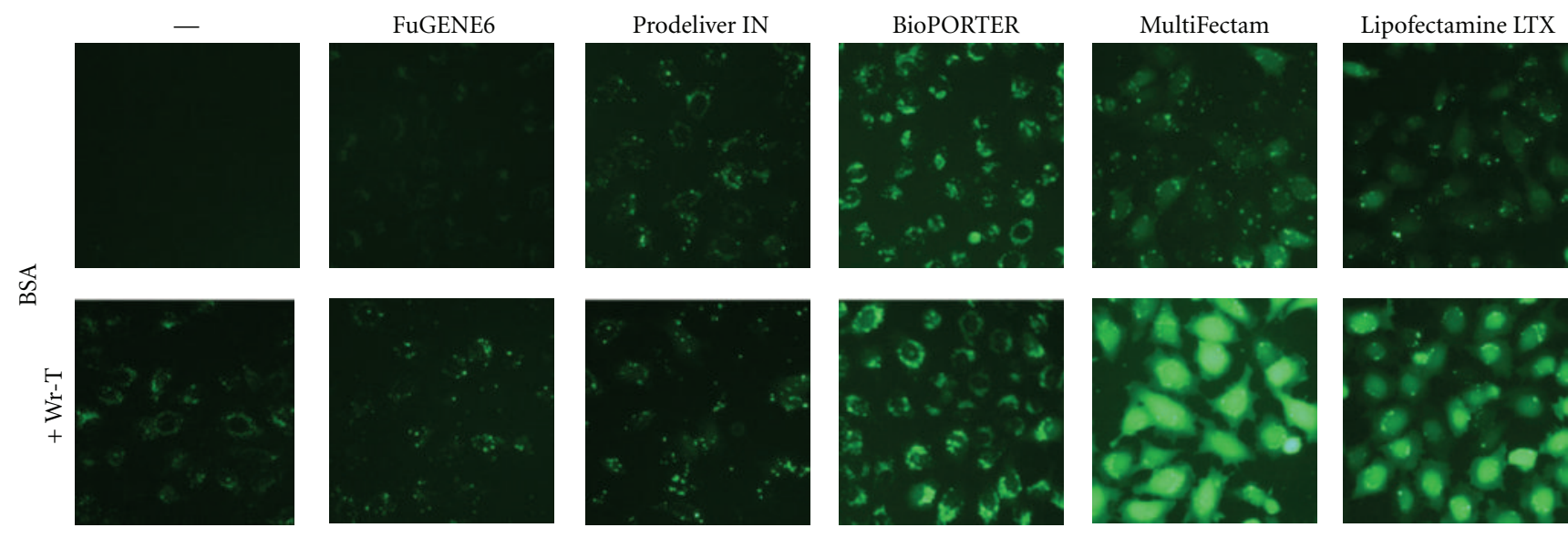

(b)

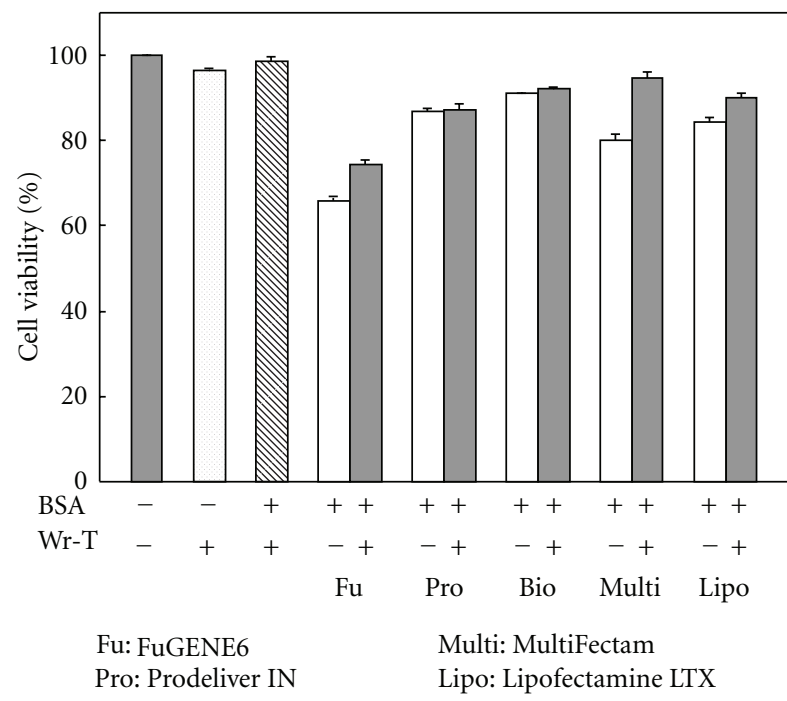

(c)

FigURE 1: Wr-T/cationic lipid reagent mixture-mediated delivery of BSA proteins into living cells. (a) Cellular localization of Wr-T/BSA complexes. The internalization of Alexa-488-labeled BSA protein and marker dyes for endocytotic vesicles into HeLa cells was analyzed by confocal laser scanning microscopy. (b) Functional properties of Wr-T/cationic lipid reagents mixture pertaining to protein transduction. HeLa cells were cotreated by BSA with Wt-T and cationic lipid reagents in serum-free medium. After incubation for $3 \mathrm{~h}$, the cells were placed in fresh medium and assessed at the same exposure time by fluorescence microscopy. (c) Cytotoxicity of Wr-T/cationic lipid reagent/cargo protein mixture. HeLa cell viability was quantified by the amount of ATP derived from metabolically active cells. Data were consistent in two repeat experiments. 


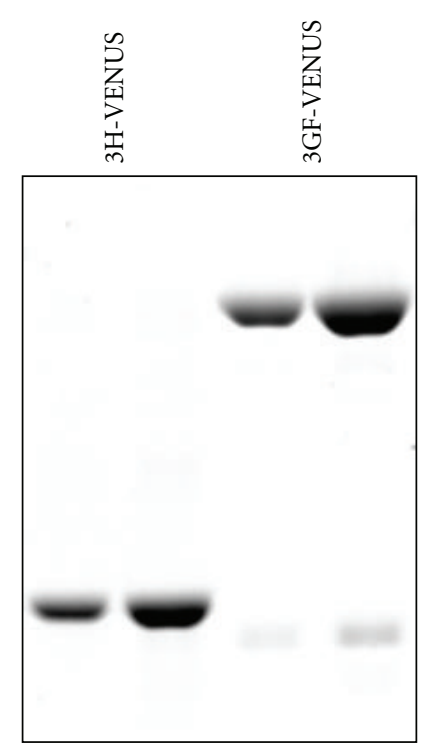

(a)

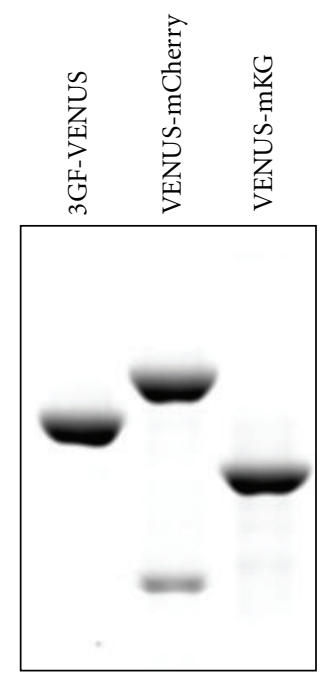

(d)
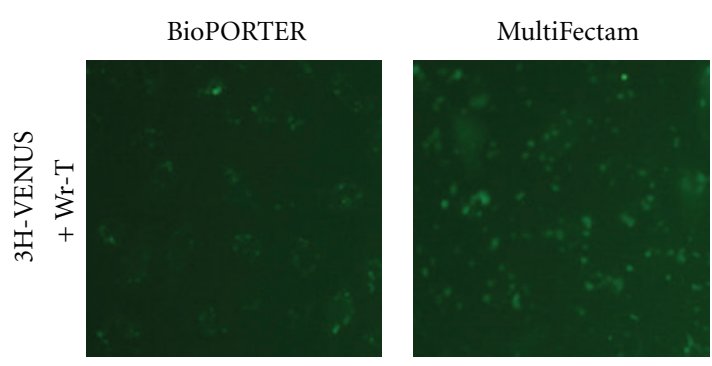

(b)
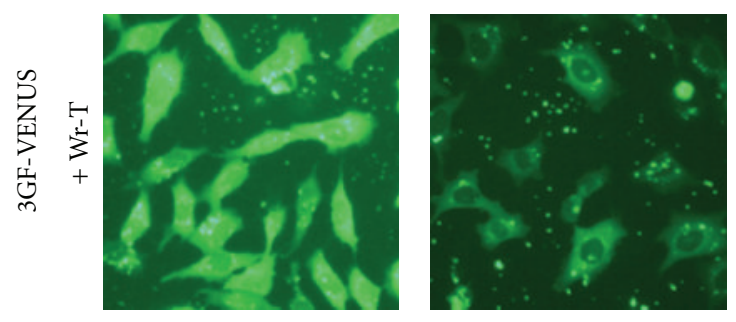

(c)
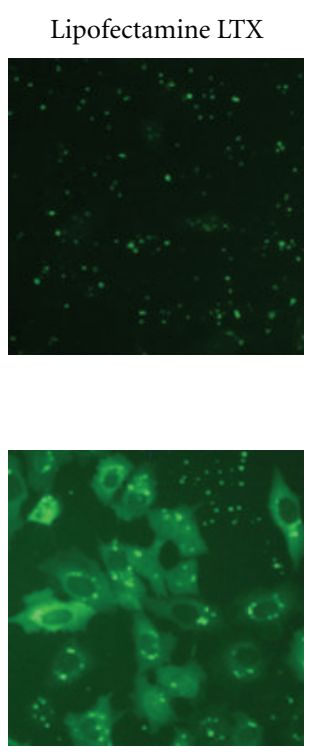

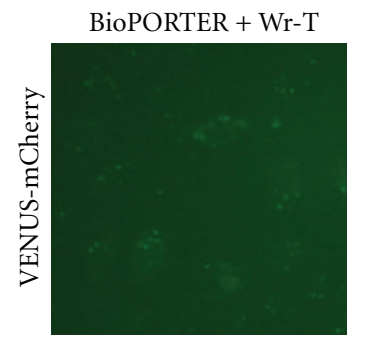

(e)

FIGURE 2: Wr-T/cationic lipid reagents mixture-mediated delivery of VENUS proteins into living cells. (a, c) Following protein purification, 3' His tag-VENUS, 3' GST and FLAG tag-VENUS, 3' mCherry and His tag-VENUS, and 3' $\mathrm{mKG}$ and His tag-VENUS were analyzed by SDSPAGE. VENUS proteins were complexed with Wr-T and cationic lipid reagents and then overlaid onto HeLa cells in a serum-free medium. Cellular localization was monitored at the same exposure time by fluorescence microscopy. (b) 3'His tag-VENUS; (c) 3'GST and FLAG tag-VENUS; (e) 3'mCherry tag-VENUS and 3' mKG tag-VENUS.

together increased the solubility and stability of expressed proteins, compared with GST tag alone.

We produced VENUS protein with GST and FLAG tag at the $\mathrm{C}$ terminus (3GF-VENUS) using a wheat germ cell-free protein synthesis system. The soluble fractions of the recombinant VENUS proteins were purified on a glutathione-Sepharose affinity column (Figure 2(a)). 3GFVENUS migrated into HeLa cells and diffused throughout the cytoplasm with a mixture of Wr-T and MultiFectam or Lipofectamine LTX (Figure 2(c)). In addition, the mixture of Wr-T and BioPORTER markedly enhanced the cytoplasmic localization of 3GF-VENUS, unlike the case of BSA. We questioned whether these results were truly dependent on GST and FLAG tag, or whether they were due to variation in molecular weight. Thus, we evaluated the delivery of two different tag-fused VENUS proteins, mCherry tag, and $\mathrm{mKG}$ tag, which have molecular weights approximately equal to those of GST and FLAG tag (Figure 2(d)). Virtually none of the Venus-mCherry and VENUS-mKG proteins with $\mathrm{Wr}-\mathrm{T}$ and BioPORTER were able to enter the cells (Figure 2(e)), and little fluorescence was observed throughout the cytoplasm. Taken together, these data indicated that 

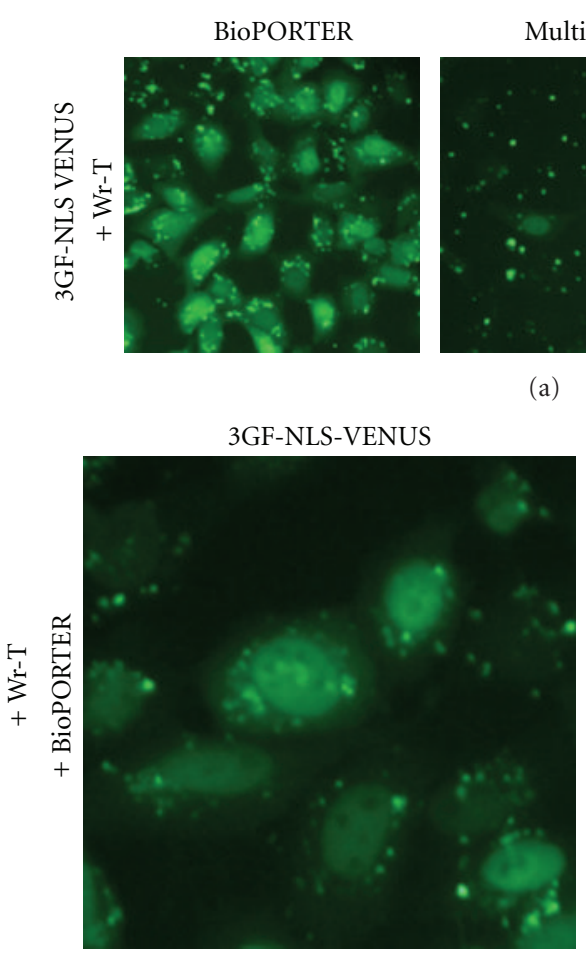

(a)
MultiFectam
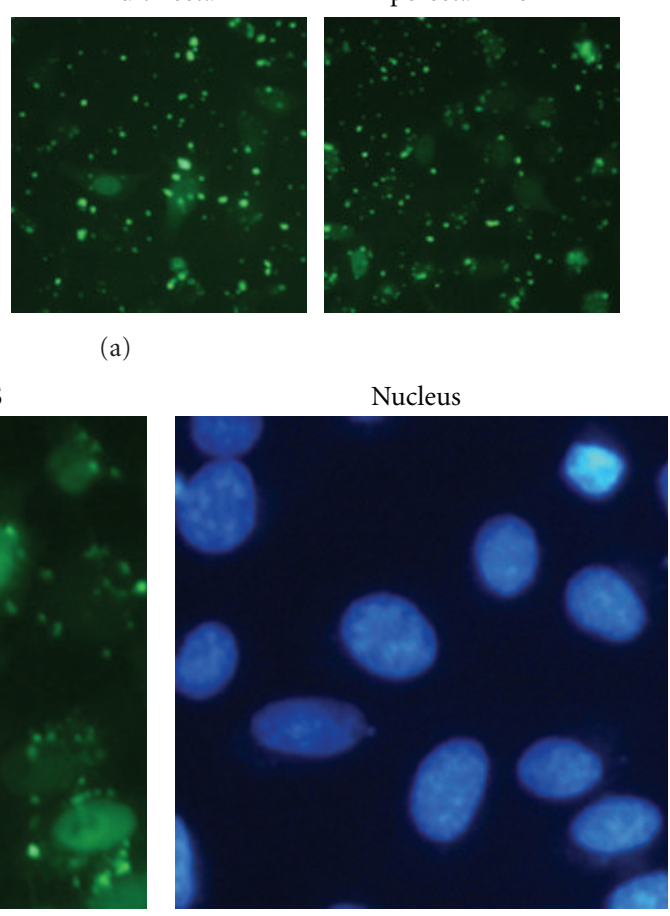

(b)

FIGURE 3: Wr-T/cationic lipid reagents mixture-mediated selective delivery of proteins into living cells. (a) 3'GST and FLAG tag-NLS VENUS proteins were incubated with Wr-T and cationic lipid reagents and then overlaid onto HeLa cells in serum-free medium for $3 \mathrm{~h}$. The cells were then washed and observed at the same exposure time by fluorescence microscopy. (b) The cells treated with Wr-T/BioPORTER mixture were counterstained with Hoescht 33342.

when VENUS protein is fused with GST and FLAG tag, they associate through noncovalent interactions with $\mathrm{Wr}$-T and BioPORTER and form stable complexes.

\subsection{GST and FLAG Tag-Fused VENUS Proteins/Wr-T/} Cationic Lipid Reagent Complexes Can be Selectively Delivered to the Nucleus by Combining NLS Peptides. To test whether a VENUS protein with nuclear transport signal (NLS) can be selectively delivered to the nucleus, we produced VENUS protein fused with NLS at the N terminus and GST and FLAG tag at the C terminus (3GF-NLS VENUS) in the same way as the 3GS-VENUS proteins. The 3GF-NLS VENUS/WrT/BioPORTER complexes were delivered to the nucleus in $>90 \%$ of Hela cells (Figures $3(\mathrm{a}$ ) and $3(\mathrm{~b})$ ). On the other hand, $<30 \%$ of the cells exhibited localization to the nucleus with Wr-T/MultiFectam or Lipofectamine LTX complexes. Similar results were shown in the case of MRC5 cells (Figure S1B of supplementary material available at doi: 10.1155/2011/872065). We were unable to detect nuclear VENUS fluorescence with MultiFectam or Lipofectamine LTX alone. Our findings showed that GST and FLAG tagfused cargo proteins can be selectively delivered to the nucleus by using NLS peptides with Wr-T and cationic lipid reagents. Furthermore, our results imply that by fusing high stability proteins such as GST and FLAG tag, cargo proteins agglutinated or not associated with Wr-T and cationic lipid reagents could form stable complexes with them and be delivered into the target organelles.

In conclusion, we described a strategy for the delivery of full-length proteins into living cells, based on association with Wr-T and cationic lipid reagents. This allowed the delivery of distinct proteins into the living cells and escape from the endosomal vesicles, without the need for crosslinking or denaturation steps. This strategy is expected to be a useful tool for studying the role of proteins and protein-protein interactions in living cells, as well as cell reprogramming using recombinant proteins. This strategy may also be a potential clinical tool for tissue engineering and regenerative medicine.

\section{References}

[1] H. Nagahara, A. M. Vocero-Akbani, E. L. Snyder et al., "Transduction of full-length TAT fusion proteins into mammalian cells: TAT-p27(Kip1) induces cell migration," Nature Medicine, vol. 4, no. 12, pp. 1449-1452, 1998.

[2] D. Derossi, A. H. Joliot, G. Chassaing, and A. Prochiantz, "The third helix of the Antennapedia homeodomain translocates through biological membranes," Journal of Biological Chemistry, vol. 269, no. 14, pp. 10444-10450, 1994.

[3] S. M. Fuchs and R. T. Raines, "Pathway for polyarginine entry into mammalian cells," Biochemistry, vol. 43, no. 9, pp. 24382444, 2004. 
[4] M. Fretz, J. Jin, R. Conibere et al., "Effects of $\mathrm{Na}^{+} / \mathrm{H}^{+}$ exchanger inhibitors on subcellular localisation of endocytic organelles and intracellular dynamics of protein transduction domains HIV-TAT peptide and octaarginine," Journal of Controlled Release, vol. 116, no. 2, pp. 247-254, 2006.

[5] J. S. Wadia, R. V. Stan, and S. F. Dowdy, "Transducible TATHA fusogenic peptide enhances escape of TAT-fusion proteins after lipid raft macropinocytosis," Nature Medicine, vol. 10, no. 3, pp. 310-315, 2004.

[6] T. Sugita, T. Yoshikawa, Y. Mukai et al., "Comparative study on transduction and toxicity of protein transduction domains," The British Journal of Pharmacology, vol. 153, no. 6, pp. 11431152, 2008.

[7] M. Kaplan, J. S. Wadia, and S. F. Dowdy, "Cationic TAT peptide transduction domain enters cells by macropinocytosis," Journal of Controlled Release, vol. 102, no. 1, pp. 247-253, 2005.

[8] C. Pan, B. Lu, H. Chen, and C. E. Bishop, "Reprogramming human fibroblasts using HIV-1 TAT recombinant proteins OCT4, SOX2, KLF4 and c-MYC," Molecular Biology Reports, vol. 37, no. 4, pp. 2117-2124, 2010.

[9] M. Lundberg and M. Johansson, "Is VP22 nuclear homing an artifact?" Nature Biotechnology, vol. 19, no. 8, pp. 713-714, 2001.

[10] E. Kondo, M. Seto, K. Yoshikawa, and T. Yoshino, "Highly efficient delivery of p16 antitumor peptide into aggressive leukemia/lymphoma cells using a novel transporter system," Molecular Cancer Therapeutics, vol. 3, no. 12, pp. 1623-1630, 2004.

[11] O. Boussif, F. Lezoualc'h, M. A. Zanta et al., "A versatile vector for gene and oligonucleotide transfer into cells in culture and in vivo: polyethylenimine," Proceedings of the National Academy of Sciences of the United States of America, vol. 92, no. 16, pp. 7297-7301, 1995.

[12] E. Kondo, T. Tanaka, T. Miyake et al., "Potent synergy of dual antitumor peptides for growth suppression of human glioblastoma cell lines," Molecular Cancer Therapeutics, vol. 7, no. 6, pp. 1461-1471, 2008. 

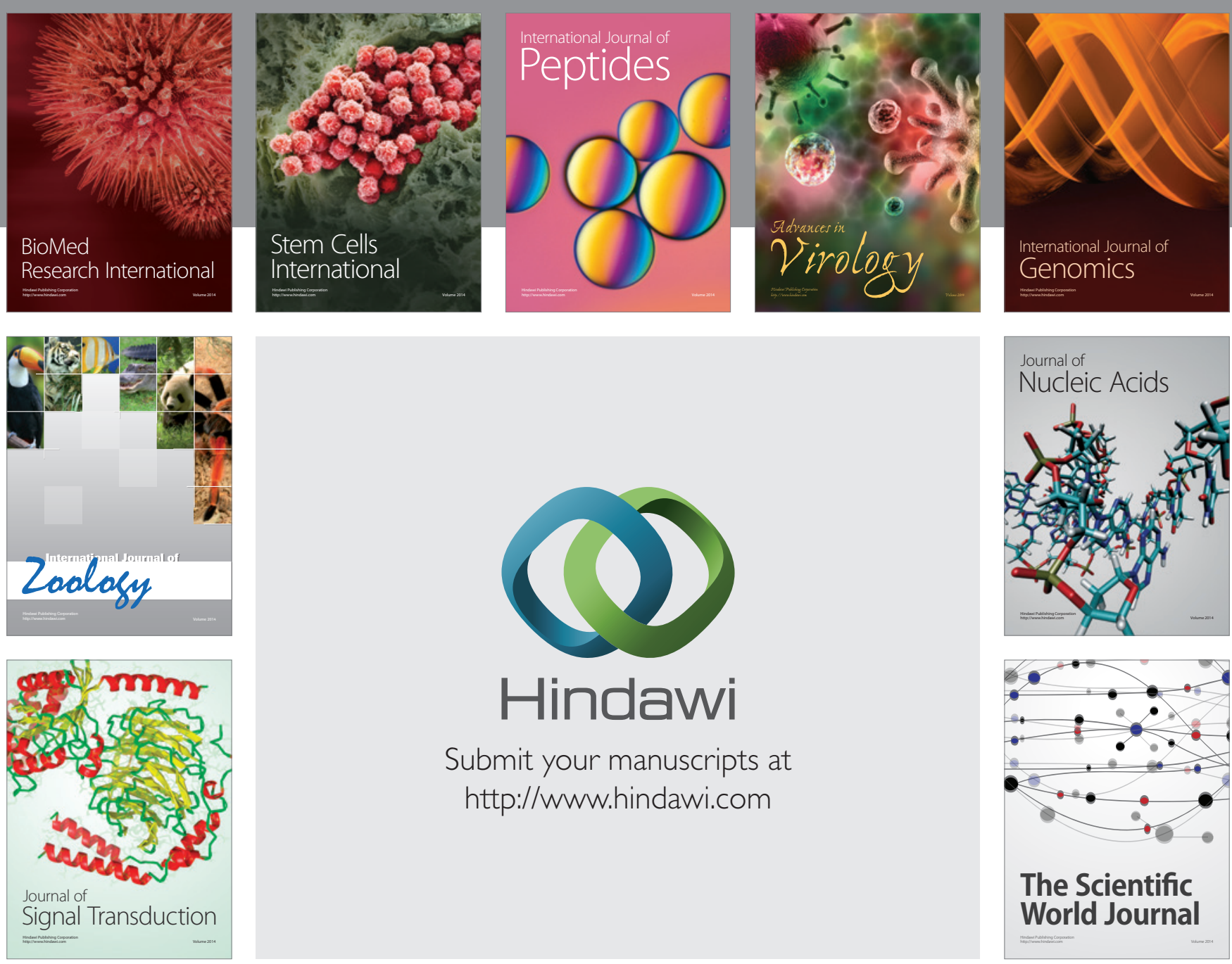

Submit your manuscripts at

http://www.hindawi.com
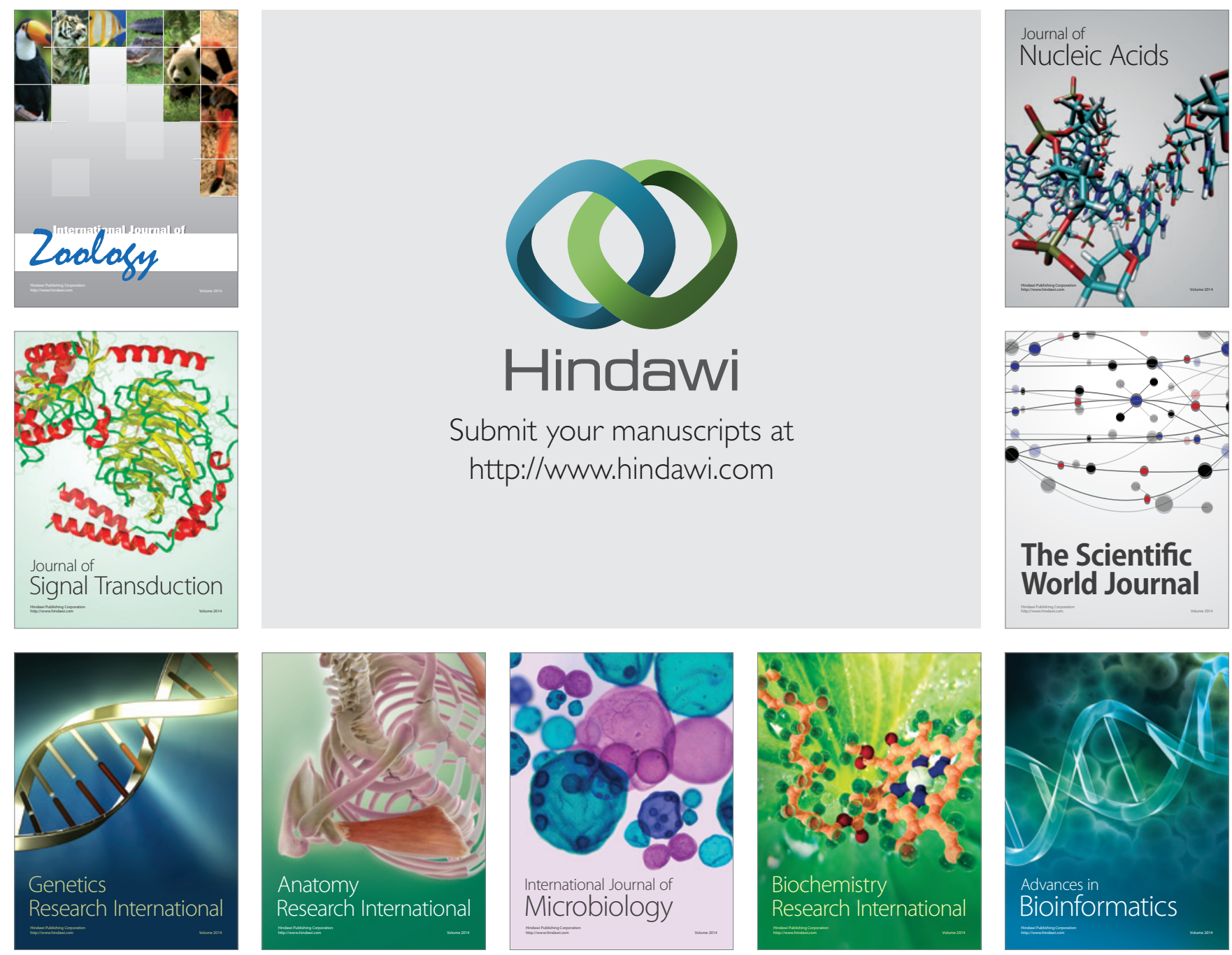

The Scientific World Journal
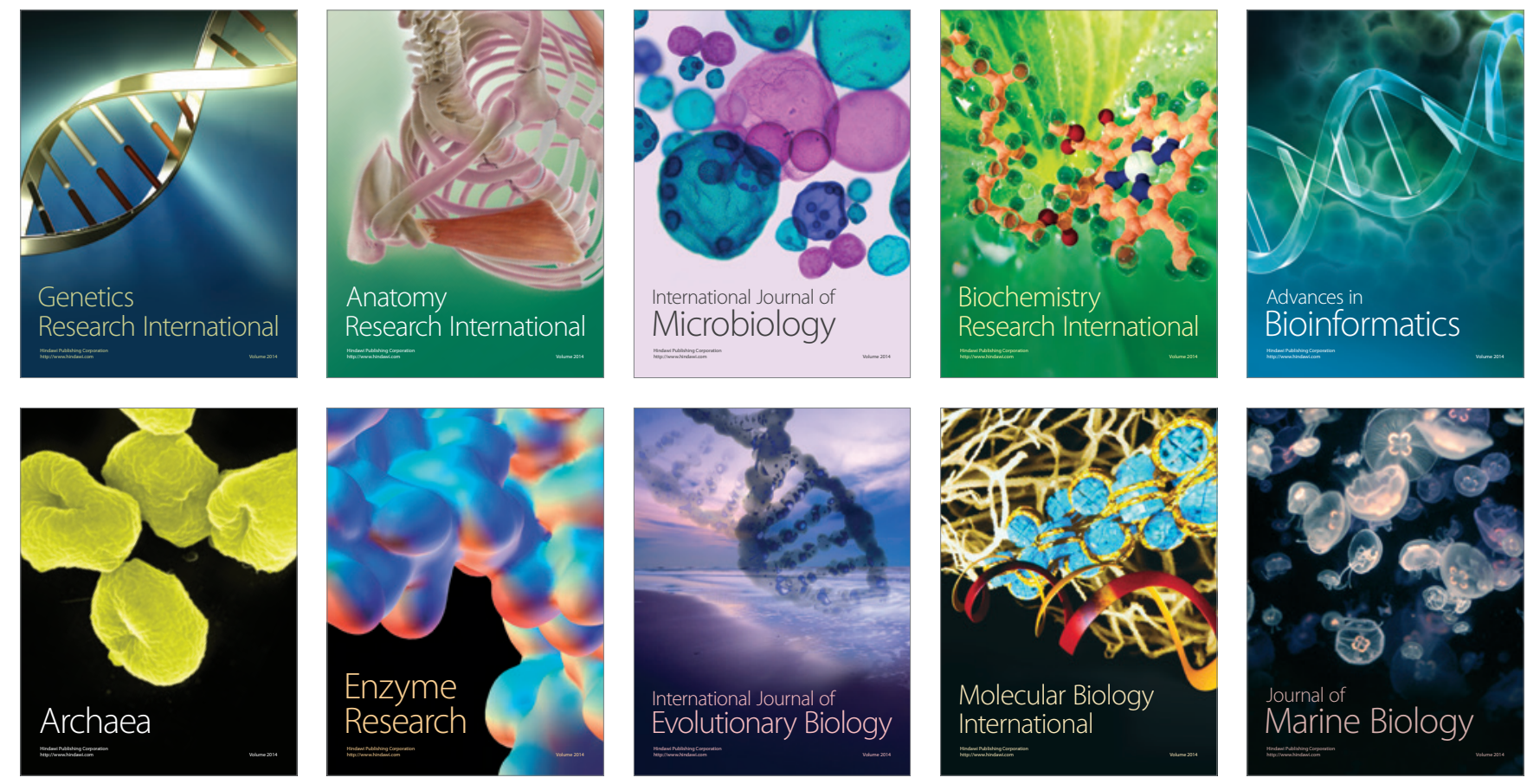